\title{
SOME TRANSFORMATIONS OF BASIC HYPERGEOMETRIC SERIES AND THEIR APPLICATIONS
}

\author{
V. K. JAIN
}

\begin{abstract}
Using Bailey's transformation, relations between basic and basic bilateral hypergeometric series are obtained. Some interesting special cases, like identities of Rogers-Ramanujan type, summation theorems for particular basic bilateral hypergeometric series ${ }_{2} \psi_{2}$, are also discussed.
\end{abstract}

1. Bailey [3], [4] used the simple transformation

$$
\sum_{n=0}^{\infty} \alpha_{n} \gamma_{n}=\sum_{n=0}^{\infty} \delta_{n} \beta_{n}
$$

where $\beta_{n}=\sum_{r=0}^{n} \alpha_{r} u_{n-r} v_{n+r}$ and $\gamma_{n}=\sum_{r=n}^{\infty} \delta_{r} u_{r-n} v_{n+r}$ to obtain transformations of basic hypergeometric series from which he deduced a number of identities of Rogers-Ramanujan type. Later on, Slater [9] used the transformation (1.1) to obtain a long list of identities of Rogers-Ramanujan type.

In $\$ 2$ of this note we use (1.1) to obtain transformations of basic hypergeometric series using some of the summation formulae which we had proved in an earlier paper [6]. In fact we prove the following three general transformations:

$$
\begin{aligned}
{ }_{4} \phi_{3}\left[\begin{array}{r}
\frac{q^{1 / 2}}{a b}, \frac{-q^{1 / 2}}{a b}, c q, d q ; q ; \frac{1}{c d} \\
-q, \frac{q}{a^{2}}, \frac{q}{b^{2}}
\end{array}\right] \\
=\Pi\left[\begin{array}{l}
\frac{q}{c}, \frac{q}{d} ; q \\
q^{2}, \frac{1}{c d}
\end{array}\right] \\
\cdot{ }_{8} \psi_{8}\left[\begin{array}{l}
q^{5 / 2},-q^{5 / 2}, c q, c q^{2}, d q, d q^{2}, a^{2} q, b^{2} q ; q^{2} ; \frac{1}{(a b c d q)^{2}} \\
q^{1 / 2},-q^{1 / 2}, \frac{q}{c}, \frac{q^{2}}{c}, \frac{q}{d}, \frac{q^{2}}{d}, \frac{q^{2}}{a^{2}}, \frac{q^{2}}{b^{2}} ; 2
\end{array}\right]
\end{aligned}
$$

Received by the editors May 11, 1978 and, in revised form, November 10, 1978.

AMS (MOS) subject classifications (1970). Primary 33A30.

Key words and phrases. Basic bilateral hypergeometric series, Bailey's transformation, identities of Rogers-Ramanujan type. 


$$
\begin{aligned}
& \text { provided }|c d|>1, c, d \neq 1, \\
& { }_{2} \phi_{2}\left[\begin{array}{l}
a q, b q ; q ; \frac{-c}{a b q} \\
-q, c
\end{array}\right]=\prod\left[\begin{array}{l}
\frac{q}{a}, \frac{q}{b} ; q \\
q^{2}, \frac{1}{a b}
\end{array}\right] \\
& \cdot{ }_{7} \psi_{7}\left[\begin{array}{l}
q^{5 / 2},-q^{5 / 2}, a q, a q^{2}, b q, b q^{2}, \frac{q^{2}}{c} ; q^{2} ; \frac{-c}{a^{2} b^{2} q^{3}} \\
q^{1 / 2},-q^{1 / 2}, \frac{q}{a}, \frac{q^{2}}{a}, \frac{q}{b}, \frac{q^{2}}{b}, c q ; 1
\end{array}\right], \quad a, b \neq 1,
\end{aligned}
$$

and

$$
\begin{aligned}
{ }_{2} \phi_{2}\left[\begin{array}{r}
c, d ; q ; \frac{-b^{2} q}{c d} \\
b \sqrt{q},-b \sqrt{q}
\end{array}\right]= & \prod\left[\begin{array}{l}
\frac{b^{2} q}{c}, \frac{b^{2} q}{d} ; q \\
b^{2} q, \frac{b^{2} q}{c d}
\end{array}\right] \\
& { }_{7} \phi_{7}\left[\begin{array}{l}
b q^{2},-b q^{2}, c, c q, d, d q, b^{2} ; q^{2} ; \frac{b^{4} q^{2}}{c^{2} d^{2}} \\
b,-b, \frac{b^{2} q}{c}, \frac{b^{2} q^{2}}{c}, \frac{b^{2} q}{d}, \frac{b^{2} q^{2}}{d}, 0
\end{array}\right],
\end{aligned}
$$

where the generalized basic and basic bilateral hypergeometric series are defined as

$$
\begin{aligned}
p+1 & \phi_{p+r}\left[\begin{array}{l}
a_{1}, a_{2}, \ldots, a_{p+1} ; q ; x \\
b_{1}, \ldots, b_{p+r}
\end{array}\right] \\
& =\sum_{n=0}^{\infty} \frac{\left[a_{1} ; q\right]_{n} \cdots\left[a_{p+1} ; q\right]_{n} x^{n}(-)^{n r} q^{m(n-1) / 2}}{[q ; q]_{n}\left[b_{1} ; q\right]_{n} \cdots\left[b_{p+r} ; q\right]_{n}}, \quad|q|<1,
\end{aligned}
$$

and

$$
\begin{aligned}
& { }_{A} \psi_{A}\left[\begin{array}{l}
a_{1}, a_{2}, \ldots, a_{A} ; q ; z \\
b_{1}, b_{2}, \ldots, b_{A} ; \lambda
\end{array}\right] \\
& =\sum_{n=-\infty}^{\infty} \frac{\left[a_{1} ; q\right]_{n} \cdots\left[a_{A} ; q\right]_{n} z^{n} q^{\lambda n(n+1) / 2}}{\left[b_{1} ; q\right]_{n} \cdots\left[b_{A} ; q\right]_{n}}, \quad|q|<1,
\end{aligned}
$$

respectively, where $[a ; q]_{n}=(1-a)(1-a q) \cdots\left(1-a q^{n-1}\right),[a ; q]_{0}=1$, and

$$
\Pi\left[\begin{array}{l}
a_{1}, \ldots, a_{r} ; q \\
b_{1}, \ldots, b_{s}
\end{array}\right]=\prod_{j=0}^{\infty} \frac{\left(1-a_{1} q^{j}\right) \cdots\left(1-a_{r} q^{j}\right)}{\left(1-b_{1} q^{j}\right) \cdots\left(1-b_{s} q^{j}\right)}
$$


The series ${ }_{p+1} \phi_{p+r}$ converges for all positive integral values of $r$ and for all $x$, except that when $r=0$, it converges only for $|x|<1$. On the other hand, the series (1.6) converges if $\lambda>0$. If $\lambda=0$ then

$$
\left|\frac{b_{1} b_{2} \cdots b_{A}}{a_{1} a_{2} \cdots a_{A}}\right|<|z|<1
$$

In the case $\lambda=0$, we drop it.

In $\$ 3$ we use (1.2)-(1.4) to deduce some new as well as known identities of Rogers-Ramanujan type with moduli 5, 6, 8, 12, 16, 20 and 24.

2. Proof of (1.2). Letting

$$
u_{s}=\frac{(-)^{s} q^{s(s+1) / 2}}{[q ; q]_{s}}, \quad v_{s}=[q ; q]_{s}, \quad \alpha_{s}=\frac{\left[\frac{q}{a^{2} b^{2}} ; q^{2}\right]_{s} q^{s}}{\left[q^{2} ; q^{2}\right]_{s}\left[\frac{q}{a^{2}} ; q\right]_{s}\left[\frac{q}{b^{2}} ; q\right]_{s}}
$$

we can evaluate $\left\langle\beta_{n}\right\rangle$ in a closed form by using the $q$-analogue of Watson's summation theorem [6] viz.

to have

$$
{ }_{4} \phi_{3}\left[\begin{array}{l}
a^{2}, b^{2},-q^{-N}, q^{-N} ; q ; q \\
a b \sqrt{q},-a b \sqrt{q}, q^{-2 N}
\end{array}\right]=\frac{\left[a^{2} q ; q^{2}\right]_{N}\left[b^{2} q ; q^{2}\right]_{N}}{\left[a^{2} b^{2} q ; q^{2}\right]_{N}\left[q ; q^{2}\right]_{N}}
$$

$$
\beta_{n}=\frac{\left[a^{2} q^{1-n} ; q^{2}\right]_{n}\left[b^{2} q^{1-n} ; q^{2}\right]_{n}(-)^{n} q^{n^{2}+n}}{\left[\frac{q}{a^{2}} ; q\right]_{n}\left[\frac{q}{b^{2}} ; q\right]_{n}(a b)^{2 n}}
$$

whereas on taking

$$
\delta_{s}=\frac{\left[q^{3} ; q^{2}\right]_{s}[c q ; q]_{s}[d q ; q]_{s}}{\left[q ; q^{2}\right]_{s}\left[\frac{q}{c} ; q\right]_{s}\left[\frac{q}{d} ; q\right]_{s}(c d q)^{s}}
$$

and using the following summation theorem

$$
{ }_{5} \phi_{5}\left[\begin{array}{l}
a, q \sqrt{a},-q \sqrt{a}, b, c ; q ; \frac{a q}{b c} \\
\sqrt{a},-\sqrt{a}, \frac{a q}{b}, \frac{a q}{c}, 0
\end{array}\right]=\prod\left[\begin{array}{l}
a q, \frac{a q}{b c} ; q \\
\frac{a q}{b}, \frac{a q}{c}
\end{array}\right]
$$

(obtained from the summation theorem [10, 3.3.1.3] by letting $d \rightarrow \infty$ ), we get

$$
\gamma_{n}=\frac{\left[q^{2} ; q\right]_{\infty}\left[\frac{1}{c d} ; q\right]_{\infty}[c q ; q]_{n}[d q ; q]_{n}}{\left[\frac{q}{c} ; q\right]_{\infty}\left[\frac{q}{d} ; q\right]_{\infty}(c d q)^{n}}
$$


378

V. K. JAIN

Making use of these values in (1.1), we get

$$
\begin{aligned}
4 \phi_{3}\left[\begin{array}{rl}
\left.\frac{q^{1 / 2}}{a b}, \frac{-q^{1 / 2}}{a b}, c q, d q ; q ; \frac{1}{c d}\right] \\
-q, \frac{q}{a^{2}}, \frac{q}{b^{2}}
\end{array}\right]=\frac{\left[\frac{q}{c} ; q\right]_{\infty}\left[\frac{q}{d} ; q\right]_{\infty}}{\left[q^{2} ; q\right]_{\infty}\left[\frac{1}{c d} ; q\right]_{\infty}} \\
\cdot \sum_{n=0}^{\infty} \frac{\left[q^{3} ; q^{2}\right]_{n}[c q ; q]_{n}[d q ; q]_{n}\left[a^{2} q^{1-n} ; q^{2}\right]_{n}\left[b^{2} q^{1-n} ; q^{2}\right]_{n}(-)^{n} q^{n^{2}}}{\left[q ; q^{2}\right]_{n}\left[\frac{q}{c} ; q\right]_{n}\left[\frac{q}{d} ; q\right]_{n}\left[\frac{q}{a^{2}} ; q\right]_{n}\left[\frac{q}{b^{2}} ; q\right]_{n}\left(a^{2} b^{2} c d\right)^{n}} \\
=\Pi\left[\begin{array}{l}
\frac{q}{c}, \frac{q}{d} ; q \\
q^{2}, \frac{1}{c d}
\end{array}\right]\left[\sum_{n=0}^{\infty} \frac{\left[q^{3} ; q^{2}\right]_{2 n}[c q ; q]_{2 n}[d q ; q]_{2 n}}{\left[q ; q^{2}\right]_{2 n}\left[\frac{q}{c} ; q\right]_{2 n}\left[\frac{q}{d} ; q\right]_{2 n}}\right. \\
\cdot \frac{\left[a^{2} q ; q^{2}\right]_{n}\left[b^{2} q ; q^{2}\right]_{n} q^{2 n^{2}}}{\left[\frac{q^{2}}{a^{2}} ; q^{2}\right]_{n}\left[\frac{q^{2}}{b^{2}} ; q^{2}\right]_{n}(a b c d)^{2 n}} \\
-\frac{1}{a b} \sum_{n=0}^{\infty} \frac{\left[q^{3} ; q^{2}\right]_{2 n+1}[c q ; q]_{2 n+1}[d q ; q]_{2 n+1}}{\left[q ; q^{2}\right]_{2 n+1}\left[\frac{q}{c} ; q\right]_{2 n+1}\left[\frac{q}{d} ; q\right]_{2 n+1}} \\
\left.\cdot \frac{\left[a^{2} ; q^{2}\right]_{n+1}\left[b^{2} ; q^{2}\right]_{n+1} q^{2 n^{2}+2 n+1}}{\left[\frac{q}{a^{2}} ; q^{2}\right]_{n+1}\left[\frac{q}{b^{2}} ; q^{2}\right]_{n+1}(a b c d)^{2 n+1}}\right] .
\end{aligned}
$$

In the last series, setting $n=-r-1$, the two series combine into a bilateral series (1.2) provided $c, d \neq 1$.

Proof of (1.3). Assuming

$$
u_{s}=\frac{q^{s^{2} / 2}}{[q ; q]_{s}}, \quad v_{s}=[q ; q]_{s}, \quad \alpha_{s}=\frac{(-c)^{s} q^{s(s-2) / 2}}{\left[q^{2} ; q^{2}\right]_{s}[c ; q]_{s}}
$$

and using the $q$-analogue of the Bailey's theorem [1] viz.

$$
{ }_{2} \phi_{2}\left[\begin{array}{l}
a, \frac{q}{a} ; q ;-b \\
-q, b
\end{array}\right]=\frac{\left[a b ; q^{2}\right]_{\infty}\left[\frac{b q}{a} ; q^{2}\right]_{\infty}}{[b ; q]_{\infty}}
$$

we get

$$
\beta_{n}=\frac{q^{n^{2} / 2}\left[c q^{-n} ; q^{2}\right]_{\infty}\left[c q^{1+n} ; q^{2}\right]_{\infty}}{[c ; q]_{\infty}}
$$

On the other hand setting

$$
\delta_{s}=\frac{\left[q^{3} ; q^{2}\right]_{s}[a q ; q]_{s}[b q ; q]_{s}(-)^{s}}{\left[q ; q^{2}\right]_{s}\left[\frac{q}{a} ; q\right]_{s}\left[\frac{q}{b} ; q\right]_{s}(a b \sqrt{q})^{s}}
$$


we obtain by using (2.3), $\left\langle\gamma_{n}\right\rangle$ in the form

$$
\gamma_{n}=\frac{\left[q^{2} ; q\right]_{\infty}\left[\frac{1}{a b} ; q\right]_{\infty}[a q ; q]_{n}[b q ; q]_{n}(-)^{n}}{\left[\frac{q}{a} ; q\right]_{\infty}\left[\frac{q}{b} ; q\right]_{\infty}(a b \sqrt{q})^{n}} .
$$

Using these values in (1.1) and proceeding as in the proof of (1.2), we get (1.3).

Proof of (1.4). Setting,

$$
u_{s}=\frac{q^{s^{2} / 2}}{[q ; q]_{s}}, \quad v_{s}=\left[b^{2} ; q\right]_{s}, \quad \alpha_{s}=\frac{(-)^{s} q^{s^{2} / 2}}{[q ; q]_{s}\left[b^{2} q ; q^{2}\right]_{s}}
$$

and using the $q$-analogue of Gauss' second summation theorem [1] viz.

$$
{ }_{2} \phi_{2}\left[\begin{array}{l}
a^{2}, b^{2} ; q ;-q \\
a b \sqrt{q},-a b \sqrt{q}
\end{array}\right]=\frac{\left[a^{2} q ; q^{2}\right]_{\infty}\left[b^{2} q ; q^{2}\right]_{\infty}}{\left[a^{2} b^{2} q ; q^{2}\right]_{\infty}\left[q ; q^{2}\right]_{\infty}},
$$

we have

$$
\beta_{n}=\frac{\left[b^{2} ; q\right]_{n}\left[b^{2} q^{1+n} ; q^{2}\right]_{\infty}\left[q^{1-n} ; q^{2}\right]_{\infty} q^{n^{2} / 2}}{[q ; q]_{n}\left[b^{2} q ; q^{2}\right]_{\infty}\left[q ; q^{2}\right]_{\infty}}
$$

However, if

$$
\delta_{s}=\frac{\left[b^{2} q^{2} ; q^{2}\right]_{s}[c ; q]_{s}[d ; q]_{s}(-)^{s}\left(b^{2} \sqrt{q}\right)^{s}}{\left[b^{2} ; q^{2}\right]_{s}\left[\frac{b^{2} q}{c} ; q\right]_{s}\left[\frac{b^{2} q}{d} ; q\right]_{s}(c d)^{s}}
$$

in view of (2.3) we get

$$
\gamma_{n}=\frac{\left[b^{2} q ; q\right]_{\infty}\left[\frac{b^{2} q}{c d} ; q\right]_{\infty}[c ; q]_{n}\left(-b^{2} \sqrt{q}\right)^{n}[d ; q]_{n}}{\left[\frac{b^{2} q}{c} ; q\right]_{\infty}\left[\frac{b^{2} q}{d} ; q\right]_{\infty}(c d)^{n}}
$$

Substituting these values in (1.1), we get (1.4) on observing that the odd part of right-hand side adds up to zero. It is of interest to note that (1.4) is a limiting case of the following more general transformation connecting a terminating Saalschützian ${ }_{5} \phi_{4}$ on the base $q$ and a well-poised ${ }_{10} \phi_{9}$ on the base $q^{2}$ (this result is very reminiscent of the Watson's $q$-analogue of Whipple's theorem [11] which expresses a terminating Saalschützian ${ }_{4} \phi_{3}$ on the base $q$ into a well-poised ${ }_{8} \phi_{7}$ on the base $q$ ):

$$
\begin{aligned}
s_{s} \phi_{4}\left[\begin{array}{l}
c, d, \sqrt{e},-\sqrt{e}, q^{-N} ; q ; q \\
e, b \sqrt{q},-b \sqrt{q}, \frac{c d}{b^{2}} q^{-N}
\end{array}\right]=\frac{\left[\frac{b^{2} q}{c} ; q\right]_{N}\left[\frac{b^{2} q}{d} ; q\right]_{N}}{\left[b^{2} q ; q\right]_{N}\left[\frac{b^{2} q}{c d} ; q\right]_{N}} \\
\cdot{ }_{10 \phi_{9}}\left[\begin{array}{l}
b^{2}, b q^{2},-b q^{2}, c, c q, d, d q, \frac{b^{2} q}{e}, q^{-N}, q^{-N+1} ; q^{2} ; \frac{b^{4} e q^{2+2 N}}{c^{2} d^{2}} \\
b,-b, \frac{b^{2} q^{2}}{c}, \frac{b^{2} q}{c}, \frac{b^{2} q^{2}}{d}, \frac{b^{2} q}{d}, e q, b^{2} q^{2+N}, b^{2} q^{1+N}
\end{array}\right] .
\end{aligned}
$$


The proof of (2.12) proceeds along the lines of that of (1.4) except in this case we use the $q$-analogue of Watson's summation theorem [2] (instead of $q$-analogue of Gauss' second summation theorem (2.9)) and the summation theorem for the well-poised ${ }_{6} \phi_{5}[10,3.3 .1 .4]$ (instead of using (2.3)) for evaluating $\left\langle\beta_{n}\right\rangle$ and $\left\langle r_{n}\right\rangle$ respectively.

3. We begin this section by noting that if in (1.2) $a, b \rightarrow 0$, the series on the left-hand side may be summed by the $q$-analogue of Gauss' theorem [10, 3.3.2.5] to yield

$$
\begin{gathered}
\frac{[q ; q]_{\infty}\left[-\frac{1}{c} ; q\right]_{\infty}\left[-\frac{1}{d} ; q\right]_{\infty}\left[\frac{1}{c d} ; q\right]_{\infty}}{[-q ; q]_{\infty}\left[\frac{q}{c} ; q\right]_{\infty}\left[\frac{q}{d} ; q\right]_{\infty}\left[-\frac{1}{c d q} ; q\right]_{\infty}}=\sum_{n=-\infty}^{\infty} \frac{\left(1-q^{4 n+1}\right)[c q ; q]_{2 n}[d q ; q]_{2 n}}{\left[\frac{q}{c} ; q\right]_{2 n}\left[\frac{q}{d} ; q\right]_{2 n}(c d q)^{2 n}} \\
=\sum_{n=-\infty}^{\infty} \frac{[c q ; q]_{2 n}[d q ; q]_{2 n}}{\left[\frac{q}{c} ; q\right]_{2 n}\left[\frac{q}{d} ; q\right]_{2 n}(c d q)^{2 n}}-\sum_{n=-\infty}^{\infty} \frac{[c q ; q]_{2 n}[d q ; q]_{2 n} q^{2 n+1}}{\left[\frac{q}{c} ; q\right]_{2 n}\left[\frac{q}{d} ; q\right]_{2 n}(c d)^{2 n}} \\
=\sum_{n=-\infty}^{\infty} \frac{[c q ; q]_{n}[d q ; q]_{n}\left(-\frac{1}{c d q}\right)^{n}}{\left[\frac{q}{c} ; q\right]_{n}\left[\frac{q}{d} ; q\right]_{n}}
\end{gathered}
$$

provided $|q|<|c d|<\left|c^{2} d^{2} q\right|, c, d \neq 1$.

Next, we deduce some identities of Rogers-Ramanujan type from the transformations proved in $\$ 2$. The technique adopted is to specialize the parameters in the transformation in such a way that the right-hand side may be summed by Jacobi's triple product identity $[8,(1.1)]$ :

$$
\sum_{n=-\infty}^{\infty}(-)^{n} x^{a n^{2}} z^{b n}=\prod_{n=1}^{\infty}\left(1-x^{(2 n-1) a} z^{b}\right)\left(1-x^{(2 n-1) a} z^{-b}\right)\left(1-x^{2 n a}\right)
$$

For instance, considering (1.2) and setting $c, d \rightarrow \infty, a=q^{-1 / 4}$ and $b^{2}=-q^{-1 / 2}$ one obtains the following identity of Rogers-Ramanujan type

$$
\sum_{n=0}^{\infty} \frac{\left[-q^{2} ; q^{2}\right]_{n} q^{n^{2}+n}}{[q ; q]_{2 n+1}}=\prod_{n \neq 0,3,9(\bmod 12)}\left(1-q^{n}\right)^{-1}
$$

On the other hand, in (1.2) letting $b, d \rightarrow \infty, a=q^{-1 / 4}, c=-q^{-1 / 2}$ and replacing $q$ by $q^{2}$, we get,

$$
\begin{aligned}
& {\left[q^{4} ; q^{2}\right]_{\infty} \sum_{n=0}^{\infty} \frac{\left[-q ; q^{2}\right]_{n} q^{n^{2}+2 n}}{\left[q^{4} ; q^{4}\right]_{n}\left[q^{3} ; q^{2}\right]} } \\
&=[q ; q]_{\infty}\left[-q^{3} ; q^{2}\right]_{\infty} \prod_{n \neq 0,5,15(\bmod 20)}\left(1-q^{n}\right)^{-1}
\end{aligned}
$$


Next, in (1.2) letting $a, b \rightarrow \infty, c=q^{-1 / 2}, d=-q^{-1 / 2}$ we get

$$
\begin{aligned}
& {\left[q^{2} ; q^{2}\right]_{\infty} \sum_{n=0}^{\infty} \frac{\left[q ; q^{2}\right]_{n}(-q)^{n}}{\left[q^{2} ; q^{2}\right]_{n}} } \\
& \quad=\left[q ; q^{2}\right]_{\infty} \prod_{n=1}^{\infty}\left(1+q^{8 n-2}\right)\left(1+q^{8 n-6}\right)\left(1-q^{8 n}\right) .
\end{aligned}
$$

Furthermore, (1.2) on letting $d \rightarrow \infty, c=-1, a=q^{1 / 4}, b^{2}=-q^{1 / 2}$ and replacing $q$ by $q^{2}$, would yield

$$
\frac{\left[q^{2} ; q^{2}\right]_{\infty}}{\left[-q^{2} ; q^{2}\right]_{\infty}} \sum_{n=0}^{\infty} \frac{\left[-1 ; q^{4}\right]_{n} q^{n^{2}+n}}{\left[q^{2} ; q^{4}\right]_{n}\left[q^{2} ; q^{2}\right]_{n}}=\prod_{n=1}^{\infty}\left(1-q^{16 n-8}\right)^{2}\left(1-q^{16 n}\right)
$$

whereas for $d \rightarrow \infty, a=q^{1 / 4}, b=q^{-1 / 4}, c=-q^{-1 / 2}$ and $q$ replaced by $q^{2},(1.2)$ yields

$$
\begin{aligned}
{\left[q^{2} ; q^{2}\right]_{\infty} \sum_{n=0}^{\infty} \frac{\left[-q ; q^{2}\right]_{n}\left[-q ; q^{2}\right]_{n} q^{n^{2}+2 n}}{\left[q^{4} ; q^{4}\right]_{n}\left[q ; q^{2}\right]_{n+1}} } \\
\quad=\left[-q ; q^{2}\right]_{\infty} \prod_{n=1}^{\infty}\left(1+q^{16 n-4}\right)\left(1+q^{16 n-12}\right)\left(1-q^{16 n}\right) .
\end{aligned}
$$

Similarly (1.3) with $a, b \rightarrow \infty$ and $c=q^{3 / 2}$, reduces to

$$
\begin{aligned}
{[-q ; q]_{\infty} } & \sum_{n=0}^{\infty} \frac{q^{3 n^{2}+2 n}}{\left[q^{4} ; q^{4}\right]_{n}\left[q ; q^{2}\right]_{n+1}} \\
& =\prod_{n \neq 0,7,13(\bmod 20)}\left(1-q^{n}\right)^{-1}+q \prod_{n \neq 0,3,17(\bmod 20)}\left(1-q^{n}\right)^{-1} \\
& =\frac{1}{[q ; q]_{\infty}} \prod_{n=1}^{\infty}\left[1+(-)^{n} q^{5 n-1}\right]\left[1-(-)^{n} q^{5 n-4}\right]\left[1-(-)^{n} q^{5 n}\right]
\end{aligned}
$$

where the last line is written by using an identity due to Bailey [4, 4.1], see also Sears [7]. Last, if in (1.4) we make $c, d \rightarrow \infty$ and then $b=1, b=q$, we get the following two known identities (Slater [9, 2.46,2.44])

$$
\begin{aligned}
& \sum_{n=0}^{\infty} \frac{q^{n(3 n-1) / 2}}{[q ; q]_{n}\left[q ; q^{2}\right]_{n}}=\prod_{n \neq 0,4,6(\bmod 10)}\left(1-q^{n}\right)^{-1} \\
& \sum_{n=0}^{\infty} \frac{q^{3 n(n+1) / 2}}{[q ; q]_{n}\left[q ; q^{2}\right]_{n+1}}=\prod_{n \neq 0,2,8(\bmod 10)}\left(1-q^{n}\right)^{-1}
\end{aligned}
$$

It may not be out of place to mention that identities involving the sum of two or three or even more triple products can also be obtained from (1.2)-(1.4) e.g. in (1.2) 
by letting $b, d \rightarrow \infty$, we have

$$
\begin{aligned}
\frac{[q ; q]_{\infty}}{\left[\frac{q}{c} ; q\right]_{\infty}} \sum_{n=0}^{\infty} \frac{[c q ; q]_{n}(-)^{n} q^{n(n+1) / 2}}{\left[q^{2} ; q^{2}\right]_{n}\left[\frac{q}{a^{2}} ; q\right]_{n} c^{n}} \\
\quad=\sum_{n=-\infty}^{\infty} \frac{\left(1-q^{4 n+1}\right)[c q ; q]_{2 n}\left[a^{2} q ; q^{2}\right]_{n}(-)^{n} q^{5 n^{2}+n}}{\left[\frac{q}{c} ; q\right]_{2 n}\left[\frac{q^{2}}{a^{2}} ; q^{2}\right]_{n}(c a)^{2 n}}
\end{aligned}
$$

Next, taking $a=q^{1 / 4}, c=q^{-1 / 2}$ and replacing $q$ by $q^{2}$, (3.11) gives a known identity due to Slater $[9,2.16]$ viz.

$$
\begin{aligned}
{\left[q^{2} ; q^{2}\right]_{\infty} } & \sum_{n=0}^{\infty} \frac{(-)^{n} q^{n^{2}+2 n}}{\left[q^{4} ; q^{4}\right]_{n}} \\
& =\left[q ; q^{2}\right]_{\infty}[q ; q]_{\infty}\left[\prod_{n \neq 0,7,13(\bmod 20)} \prod_{q} \prod_{n \neq 0,3,17(\bmod 20)}\left(1-q^{n}\right)^{-1}\right. \\
& =\left[q ; q^{2}\right]_{\infty} \prod_{n=1}^{\infty}\left[1+\left(-q^{n}\right)^{-1}\right]
\end{aligned}
$$

whereas taking $a=q^{1 / 4}, c=-q^{-1 / 2}$ and replacing $q$ by $q^{2}(3.11)$ yields

$$
\begin{aligned}
{\left[q^{2} ; q^{2}\right]_{\infty} } & \sum_{n=0}^{\infty} \frac{\left[-q ; q^{2}\right]_{n} q^{n^{2}+2 n}}{\left[q^{4} ; q^{4}\right]_{n}\left[q ; q^{2}\right]_{n}} \\
& =\left[-q ; q^{2}\right]_{\infty}[q ; q]_{\infty}\left[\prod_{n \neq 0,7,13(\bmod 20)} \prod_{-q} \prod_{n \neq 0,3,17(\bmod 20)}\left(1-q^{n}\right)^{-1}\right. \\
& =\left[-q ; q^{2}\right]_{\infty} \prod_{n=1}^{\infty}\left[1-\left(-q^{n}\right)^{-1} q^{5 n-1}\right]\left[1+(-)^{n} q^{5 n-4}\right]\left[1-(-)^{n} q^{5 n}\right]
\end{aligned}
$$

Combining (3.12) and (3.13), one obtains identities for the triple product $\Pi_{n \neq 0,7,13(\bmod 20)}\left(1-q^{n}\right)^{-1}$ and $\Pi_{n \neq 0,3,17(\bmod 20)}\left(1-q^{n}\right)^{-1}$. It may also be observed that (3.8) and (3.12) yield a series transformation. But if we had started with the identity (3.8), we would have not been able to obtain the identities on modulus 20 which could be obtained from (3.12). 
The identity (3.11) for $a=q^{-1 / 4}, c=q^{1 / 2}$ would correspond to (cf. Bailey [5, 4.2])

$$
\begin{aligned}
{\left[q^{2} ; q^{2}\right]_{\infty} } & \sum_{n=0}^{\infty} \frac{(-)^{n} q^{n^{2}}}{\left[q^{4} ; q^{4}\right]_{n}} \\
& =\left[q ; q^{2}\right]_{\infty}[q ; q]_{\infty}\left[\prod_{n \neq 0,9,11(\bmod 20)}\left(1-q^{n}\right)^{-1}\right. \\
-q^{2} & \left.\prod_{n \neq 0,1,19(\bmod 20)}\left(1-q^{n}\right)^{-1}\right] \\
& =\left[q ; q^{2}\right]_{\infty} \prod_{n=1}^{\infty}\left[1-(-)^{n} q^{5 n-2}\right]\left[1+(-)^{n} q^{5 n-3}\right]\left[1-(-)^{n} q^{5 n}\right],
\end{aligned}
$$

and for $a=q^{-1 / 4}, c=-q^{1 / 2}$ would lead to

$$
\begin{aligned}
& {\left[q^{2} ; q^{2}\right]_{\infty} } \sum_{n=0}^{\infty} \frac{\left[-q^{3} ; q^{2}\right]_{n} q^{n^{2}}}{\left[q^{4} ; q^{4}\right]_{n}\left[q ; q^{2}\right]_{n+1}} \\
&\left.=\left[-q^{3} ; q^{2}\right]_{\infty}[q ; q]_{\infty}\right]_{n \neq 0,9,11(\bmod 20)}\left(1-q^{n}\right)^{-1} \\
&+q^{2} \prod_{n \neq 0,1,19(\bmod 20)} \prod_{+2 q} \prod_{n \neq 0,5,15(\bmod 20)}\left(1-q^{n}\right)^{-1} \\
&
\end{aligned}
$$

Identities (3.4), (3.14) and (3.15) can be combined to produce series expansions for the triple products $\Pi_{n \neq 0,1,19(\bmod 20)}\left(1-q^{n}\right)^{-1}$ and $\Pi_{n \neq 0,9,11(\bmod 20)}\left(1-q^{n}\right)^{-1}$, but for reasons of brevity, we do not mention them here.

Also, note that letting $a, b, d \rightarrow \infty$ and giving for $c$ in succession, the values $q^{-1 / 2}$ and $q^{1 / 2},(1.2)$ reduces to the following identities:

$$
\begin{aligned}
& \frac{\left[q^{2} ; q^{2}\right]_{\infty}}{\left[q ; q^{2}\right]_{\infty}} \sum_{n=0}^{\infty} \frac{\left[q ; q^{2}\right]_{n}(-)^{n} q^{n^{2}+2 n}}{\left[q^{4} ; q^{4}\right]_{n}} \\
&=\prod_{n=1}^{\infty}\left(1+q^{24 n-8}\right)\left(1+q^{24 n-16}\right)\left(1-q^{24 n}\right) \\
& \quad+q \prod_{n=1}^{\infty}\left(1+q^{24 n-4}\right)\left(1+q^{24 n-20}\right)\left(1-q^{24 n}\right) \\
&=\prod_{n=1}^{\infty}\left(1+q^{6 n-1}\right)\left(1+q^{6 n-5}\right)\left(1-q^{6 n}\right),
\end{aligned}
$$


and

$$
\begin{aligned}
\frac{\left[q^{2} ; q^{2}\right]_{\infty}}{\left[q ; q^{2}\right]_{\infty}} \sum_{n=0}^{\infty} \frac{\left[q ; q^{2}\right]_{n+1}(-)^{n} q^{n^{2}}}{\left[q^{4} ; q^{4}\right]_{n}} \\
=\prod_{n=1}^{\infty}\left(1+q^{24 n-12}\right)^{2}\left(1-q^{24 n}\right) \\
\quad-q \prod_{n=1}^{\infty}\left(1+q^{24 n-8}\right)\left(1+q^{24 n-16}\right)\left(1-q^{24 n}\right) \\
\quad-q^{2} \prod_{n=1}^{\infty}\left(1+q^{24 n-4}\right)\left(1+q^{24 n-20}\right)\left(1-q^{24 n}\right) \\
\quad+q^{3} \prod_{n=1}^{\infty}\left(1+q^{24 n}\right)\left(1+q^{24 n-24}\right)\left(1-q^{24 n}\right) \\
=\prod_{n=1}^{\infty}\left(1+q^{6 n-3}\right)^{2}\left(1-q^{6 n}\right)-q \prod_{n=1}^{\infty}\left(1+q^{6 n-5}\right)\left(1+q^{6 n-1}\right)\left(1-q^{6 n}\right) .
\end{aligned}
$$

Manipulating (3.16) with the identity obtained from it by replacing $q$ by $-q$, one obtains the two identities involving infinite products on modulus 24 .

In this connection it may be remarked that if one starts with the corresponding identity of Slater $[9,2.87]$ and proceeds as described above, one would end up with only two known identities on modulus 12 [9, (2.50) and (2.51)]. We conclude with the remark that the above listing of the identities is not exhaustive. Indeed one can get a large number of identities from the transformations discussed in \$2.

I am grateful to Dr. Arun Verma for suggesting the problem and for his help during the preparation of this paper.

\section{REFERENCES}

1. G. E. Andrews, On the q-analogue of Kummer's theorem and applications, Duke Math. J. 40 (1973), 525-528.

2. , On q-analogues of the Watson and Whipple summations, SIAM J. Math. Anal. 7 (1976), 332-336.

3. W. N. Bailey, Some identities in combinatory analysis, Proc. London Math. Soc. (2) 49 (1947), $421-435$.

4. __ Identities of the Rogers-Ramanujan type, Proc. London Math. Soc. (2) 50 (1949), 1-10.

5. On the simplification of some identities of the Rogers-Ramanujan type, Proc. London Math. Soc. (3) 1 (1951), 217-221.

6. V. K. Jain, Some transformations of basic hypergeometric functions (to appear).

7. D. B. Sears, Two identities of Bailey, Proc. London Math. Soc. (3) 1 (1951), 510-511.

8. L. J. Slater, A new proof of Roger's transformations of infinite series, Proc. London Math. Soc. (2) 53 (1951), 460-475.

9. 147-167. , Further identities of the Rogers-Ramanujan type, Proc. London Math. Soc. (2) 54 (1952),

10. __ Generalized hypergeometric functions, Cambridge Univ. Press, New York, 1966.

11. G. N. Watson, A new proof of the Rogers-Ramanujan identities, J. London Math. Soc. 4 (1929), 4-9.

Department of Mathematics, ROORKeE UnIVERstTy, ROORKeE, 247672 (U.P.) INDIA 